EPJ Web of Conferences 73, 03012 (2014)

DOI: $10.1051 /$ epjconf/20147303012

(C) Owned by the authors, published by EDP Sciences, 2014

\title{
Study of light mesons with WASA-at-COSY
}

\author{
Elisabetta Prencipe $^{\mathrm{a}}$ on behalf of the WASA-at-COSY Collaboration \\ Forschungszentrum Jülich GmbH, 52425 Jülich, Germany
}

\begin{abstract}
The WASA detector, operating at the COSY facility in Jülich (Germany) has been collecting data since 2007. The experiment allows to perform studies of light mesons, such as $\pi^{0}, \eta$ and $\omega$ rare decay processes, in order to perform precise measurements of branching ratios, determine Dalitz plot parameters, test symmetry and symmetry breaking, and evaluate transition form factors. In the experiments a proton or deuteron beam impinged on a pellet target of hydrogen or deuterium, which allows the reactions proton-proton $(p p)$ or proton-deuteron $(p d)$. A high-statistics sample of $\eta$ mesons has been collected: in the reaction $p d \rightarrow{ }^{3} \mathrm{He} \eta, 3 \times 10^{7} \eta$ mesons were tagged at a beam energy of $1.0 \mathrm{GeV}$, while $5 \times 10^{8} \eta$ mesons were produced in the reaction $p p \rightarrow p p \eta$ at $1.4 \mathrm{GeV}$. This corresponds to the production of $10 \eta / \mathrm{s}$ and $100 \eta / \mathrm{s}$, respectively, for the two reaction processes. In the $p p$ dataset a higher background level is found compared to the $p d$ data set. In both cases, we identify the $\eta$ mesons by means of the missing mass derived from the recoil particles. A kinematic fit largely rejects the background in our analysis. The advantage in using the $p p$ dataset is that the production of $\eta$ mesons is almost a factor of 10 higher than in the $p d$ fusion to ${ }^{3} \mathrm{He}$. As we plan to measure the branching ratios of very rare processes, high statistics is needed. A summary of the recent activity on the study of light mesons with WASA-at-COSY here is given.
\end{abstract}

\section{Introduction}

The experiment WASA [1] (Wide Angle Shower Apparatus), located in Jülich (Germany), is a largeacceptance detector for charged and neutral particles. It was installed at the Cooler Synchrotron COSY in summer 2006 and has successfully taken data. The installation of WASA at COSY has significantly enlarged the possibilities of the COSY facility [2]. The design of this detector allows the study of light mesons produced in different reactions: proton-proton $(p p)$ and proton-deuteron $(p d)$.

The study of light mesons provides an ideal laboratory for:

1. precision tests and experimental input to ChPT (Chiral perturbation theory);

2. tests of symmetry and symmetry breaking;

3. studies of rare decays of $\eta, \pi^{0}$ and $\omega$;

4. determination of Dalitz plot parameters;

5. study of transition form factor;

6. search for new physics beyond the Standard Model (SM).

\footnotetext{
ae-mail: e.prencipe@fz-juelich.de
}

This is an Open Access article distributed under the terms of the Creative Commons Attribution License 4.0, which permits unrestricted use, distribution, and reproduction in any medium, provided the original work is properly cited. 
Table 1. $\pi^{0}$ decays under study at WASA.

\begin{tabular}{lll}
\hline Decay & BR (PDG) & Physics case \\
\hline$\pi^{0} \rightarrow e^{+} e^{-\gamma}$ & $(1.174 \pm 0.035) \times 10^{-2}$ & physics beyond the SM: U boson \\
$\pi^{0} \rightarrow e^{+} e^{-}$ & $(6.46 \pm 0.33) \times 10^{-8}$ & physics beyond the SM \\
\hline
\end{tabular}

Table 2. $\eta$ decays under study at WASA.

\begin{tabular}{lll}
\hline Decay & BR (PDG) & Physics case \\
\hline$\eta \rightarrow \pi^{+} \pi^{-} \pi^{0}$ & $(22.92 \pm 0.28) \times 10^{-2}$ & ChPT test: Dalitz plot parameters \\
$\eta \rightarrow \pi^{+} \pi^{-} \gamma$ & $(4.22 \pm 0.08) \times 10^{-2}$ & ChPT test: box anomaly and BR calculation \\
$\eta \rightarrow e^{+} e^{-} \gamma$ & $(6.9 \pm 0.4) \times 10^{-3}$ & Transition form factor and BR calculation \\
$\eta \rightarrow \pi^{+} \pi^{-} e^{+} e^{-}$ & $(2.68 \pm 0.11) \times 10^{-4}$ & CP test of SM and BR calculation \\
$\eta \rightarrow e^{+} e^{-} e^{+} e^{-}$ & $(2.40 \pm 0.22) \times 10^{-5}$ & Double transition form factor and BR calculation \\
$\eta \rightarrow \pi^{0} e^{+} e^{-}$ & $<4 \times 10^{-5}$ & C test of SM and BR calculation \\
$\eta \rightarrow e^{+} e^{-}$ & $<5.6 \times 10^{-6}$ & Physics beyond SM \\
\hline
\end{tabular}

Table 3. $\omega$ decays under study at WASA.

\begin{tabular}{lll}
\hline Decay & BR (PDG) & Physics case \\
\hline$\omega \rightarrow \pi^{+} \pi^{-} \pi^{0}$ & $(89.2 \pm 0.7) \times 10^{-2}$ & Dalitz plot parameters \\
$\omega \rightarrow \pi^{0} \gamma$ & $(8.28 \pm 0.28) \times 10^{-2}$ & BR calculation \\
$\omega \rightarrow \pi^{+} \pi^{-}$ & $\left(1.53_{-0.13}^{+0.11}\right) \times 10^{-2}$ & $\rho$ - $\omega$ mixing \\
$\omega \rightarrow \pi^{0} e^{+} e^{-}$ & $(7.7 \pm 0.6) \times 10^{-4}$ & Transition form factor \\
\hline
\end{tabular}

In this report we summarize the status-of-art with WASA-at-COSY and we will give a short description of the analysis technique for several ongoing analyses. The preliminary results obtained at WASA are mainly with the $p d$ data set. However, an update of those measurements on the full $p p$ data set is ongoing, as the larger statistics available on the $p p$ data set is expected to deliver more precise results and a cross check with the previous measurements. In the Tables 1, 2 and 3 an overview of the decay under study with WASA-at-COSY are shown: the branching ratio (BR) measurements here reported are taken from the Particle Data Group (PDG) [3].

\section{The detector}

WASA is a fixed-target detector [4]. A proton or deuteron beam is scattered against to a frozen hydrogen or deuterium pellet target. The detector consists of three main components:

1. the Forward Detector (FD), covering scattering angles in the range of [3-18] degrees, used for tagging and triggering of meson production;

2. the Central Detector (CD), used for measuring meson decay products;

3. the pellet target system.

The target beam consists of 20-30 $\mu$ m diameter pellets of hydrogen, providing an areal target density in the order of $10^{15}$ atoms $/ \mathrm{cm}^{2}$. The diameter of the pellet beam is approximatively $3.8 \mathrm{~mm}$. The CD surrounds the interaction region and is designed to detect and identify photons, electrons, and charged pions. It consists of an inner drift chamber (MDC), a superconducting solenoid providing the magnetic field for momentum determination, a barrel of thin plastic scintillators (PS) for particle identification and triggering, and an electromagnetic calorimeter. The amount of structural material is kept to a minimum to reduce the amount of secondary interactions outside of the detector sensitive volumes. The beryllium beam pipe (diameter $6 \mathrm{~cm}$ ) wall is $1.2 \mathrm{~mm}$ thick and the material of the superconducting solenoid corresponds to 0.18 radiation lengths. The track coordinates are provided by four sets of straw proportional chambers. Kinetic energies are reconstructed using the energy information in layers of plastic scintillators of different thickness. In addition, the signals are used for triggering. 


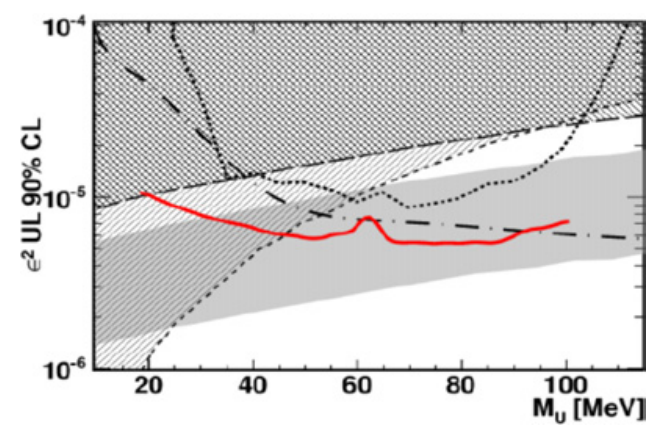

Figure 1. Summary of $90 \%$ CL upper limit for the mixing parameter $\epsilon^{2}$ from WASA-at-COSY (red solid line) compared to SINDRUM $\pi^{0} \rightarrow e^{+} e^{-} \gamma$ [11] (dotted line) and recent combined KLOE $\phi \rightarrow \eta e^{+} e^{-}$[8] (dashed dotted line) upper limit. The long respectively short dashed lines (and corresponding hatched areas) are the upper limits derived from the muon and electron $g-2$ [12]. In addition, the grey area represents the $\pm 2 \sigma$ preferred band around the present value of the muon $g-2$.

\section{3. $\pi^{0}$ decays}

The 2 decay processes under exam at WASA are summarized in Table 1, showing the BRs of those from the PDG. Table 1 highlights the interest of the physics case.

The analysis of $\pi^{0} \rightarrow e^{+} e^{-} \gamma$, in particular, was performed on $5 \times 10^{6}$ events of the $p p$ data set, which is only part of the full data sample available. The obtained results fix a new UL on the existence of the $\mathrm{U}$ boson in the process $\pi^{0} \rightarrow U \gamma, U \rightarrow e^{+} e^{-}$. The $\mathrm{U}$ boson is supposed to be a light vector boson, mixing with the photon, in this decay process. It could give possible explanation for the $511 \mathrm{keV}$ emission from the center of galaxy [5,6]. The interest in this decay channel has grown in the field of the light dark matter search [7]. The results found at WASA confirm previous experimental results [8,9] and it was published recently [10]. A new UL on the square of the $U-\gamma$ mixing strength parameter $\epsilon^{2}$ of $5 \times 10^{-6}$ at $90 \%$ confidence level (CL) was obtained in the mass range [20-100] MeV/c $\mathrm{c}^{2}$. This result, together with other recent experimental limits, significantly reduces the parameter space of the mass $M_{U}$ versus $\epsilon^{2}$, which could also explain the presently seen deviation between the SM prediction and the direct measurement of the anomalous magnetic moment of the muon $(g-2) / 2$.

\section{4. $\eta$ decays}

We are looking at several decay channels of the $\eta$ meson at WASA. In Table 2 the proposed $\eta$ decay channels and the physics case for each of those is reported. Some of the proposed decay channels have been studied in $p d$ reactions during the past years [13]; we are performing measurements of BR in $p p$ interactions now, because in $p p$ interactions we have obtained larger statistics, despite of the higher level of background observed. We can reconstruct the invariant mass of the decay products, either to analyze the missing mass of the event in the process $p p \rightarrow p p \eta, \eta \rightarrow X$, where $X$ represent every final state reported in Table 2. With the analysis of the missing mass of the event it is found an improvement in the mass resolution. In addition, the kinematic fit helps to reduce drastically the combinatorial background which affects such analyses, so preliminary results with the $p p$ data set seems to be very promising. The analysis $\eta \rightarrow e^{+} e^{-}$, indeed, was performed on the first 2 weeks of $p p$ interaction data taking. We plan to update this BR measurement on the full $p p$ data set. It could be sensitive to new physics beyond the SM. 


\section{5. $\omega$ decays}

Recently the WASA-at-COSY collaboration proposed a plan for the study of the $\omega$ decays. The decays under investigation are summarized in Table 3. Preliminary resuls were shown at this conference with both data sample, $p d$ and $p p$ ones. The study of $\omega$ decays is in progress in this moment, and techniques to improve the $\omega$ reconstruction efficiency are under investigation.

\section{Conclusion}

WASA collected a huge amount of data in both data sample, $p d$ and $p p$. Several analyses are ongoing for the study of $\pi^{0}, \eta$ and $\omega$ decays. High precision is expected in the analyses of rare decay processes on the full $p p$ data set. The experience gained from the analyses of the meson decays in $p d$ data set taught to optimize the analysis techniques; by using kinematic fit the high background level, which affects analyses with $p p$ dataset, is under control. WASA is a detector suited for studying light meson decays, and it will give contribution in understanding the box anomaly, form factors of those mesons, Dalitz parameters, physics beyond the SM and symmetry breaking phenomena. Preliminary results on several $\eta$ decays have been shown using the $p d$ data set, as results of $\mathrm{PhD}$ theses. The plan is to publish those once the analyses of $p p$ data will be completed.

\section{References}

[1] http://collaborations.fz-juelich.de/ikp/wasa/

[2] http://www.fz-juelich.de/ikp/EN/Forschung/Beschleuniger/_doc/COSY.html

[3] J. Beringer at al., Phys. Rev. D 86, 010001 (2012)

[4] H.H. Adam et al., arXiv:0411038 [nucl-ex] (2004)

[5] Y. Kahn et al., Phys. Rev. D 8, 115002 (2008)

[6] Weidenspointner et al., Nature 451, 59-162 (2008)

[7] P.A.R. Ade et al., Plank Collaboration, arXiv:1303.5062 [astro-ph] (2013)

[8] D. Babusci et al., KLOE Collaboration, Phys. Lett. B 720, 111 (2013)

[9] G. Agakishiev et al., HADES Collaboration, arXiv:1311.0216 [hep-ex] (2013)

[10] P. Adlarson et al., WASA-at-COSY Collaboration, Phys. Lett. B 726, 187-193 (2013)

[11] R. Meijer Drees et al., SINDRUM Collaboration, Phys. Rev. Lett. 68, 3845 (1992)

[12] M. Endo, K. Hamaguchi, G. Mishima, Phys. Rev. D 86, 095029 (2012)

[13] P. Adlarson et al., Phys. Lett. B 707, 243-249 (2012); C. Adolph et al., Phys. Lett. B 677, 24-29 (2009); D. Coderre, PoS CD12, 063 (2013); M. Hodana et. al, EPJ Web Conf. 37, 09017 (2012); M. Berlowski, Int. J. Mod. Phys. A 26, 630-631 (2011) 